\section{Thermophilic biohydrogen production from palm oil mill effluent: Effect of immobilized cells on granular activated carbon in fluidized bed reactor}

By: Jamali, NS (Jamali, Nur Syakina) ${ }^{[1]}$; Rashidi, NFD (Rashidi, Nur Farahana Dzul) ${ }^{[1]}$; Jahim, JM (Jahim, Jamaliah Md) ${ }^{[2]}$; O-Thong, S (O-Thong, Sompong) ${ }^{[3]}$; Jehlee, A (Jehlee, Aminee) ${ }^{[3]}$; Engliman, NS (Engliman, Nurul Sakinah) ${ }^{[4]}$

\section{FOOD AND BIOPRODUCTS PROCESSING}

Volume: 117 Pages: $231-240$

DOI: $10.1016 / \mathrm{j} . \mathrm{fbp} .2019 .07 .012$

Published: SEP 2019

Document Type: Article

View Journal Impact

\section{Abstract}

In this study, the performance of immobilized cells on granular activated carbon (GAC) for thermophilic biohydrogen production is determined using POME as a fermentation substrate. The immobilized cells are formed at different $\mathrm{pH}$ medium using sugar composition characterized in the POME. The $\mathrm{pH} 6$ revealed the optimum $\mathrm{pH}$ used for biofilm development with $\mathrm{HPR}$ of $2.8 \mathrm{mmol} \mathrm{H}-2 / \mathrm{L} \mathrm{h}$. The effect of sugar utilization by the immobilized cells on GAC are determined at different sugar concentration using the Monod model prior validated the performance of the cells in the fluidized bed reactor (FBR). From the model, $0.316+/-0.013 \mathrm{~h}(-1)$ of maximum specific growth rate was obtained at $20 \mathrm{~g} / \mathrm{L}$ sugar used and was keep increasing to the maximum of $30 \mathrm{~g} / \mathrm{L}$ of sugar used with HPR 2.6-2.8 mmol H-2/L h. Lastly, the POME-enriched nutrients are used as the carbon source in the fluidized bed reactor (FBR). The highest HPR obtained was at HRT $12 \mathrm{~h},(5.2 \mathrm{mmol} \mathrm{H}-2 / \mathrm{L} \mathrm{h})$ and HY of $1.24 \mathrm{~mol} \mathrm{H}-2 / \mathrm{mol}$ sugar. The screening of the microbial population by DGGE revealed that the Thermoanaer-obacterium thermosaccharolyticum sp. was dominant for all the HRTs, thereby indicating that this bacterium is resilient towards environmental disturbances. (C) 2019 Institution of Chemical Engineers. Published by Elsevier B.V. All rights reserved.

\section{Keywords}

Author Keywords: Biohydrogen production; Immobilized cells; Activated carbon; Palm oil mill effluent; Fluidized bed reactor KeyWords Plus: CONTINUOUS HYDROGEN-PRODUCTION; BACTERIAL COMMUNITY; THERMOANAEROBACTERIUM-THERMOSACCHAROLYTICUM; BIOFILM FORMATION; SLUDGE; GLUCOSE; WASTE

\section{Author Information}

Reprint Address: Jamali, NS (reprint author)

+ Univ Putra Malaysia, Fac Engn, Dept Chem \& Environm Engn, Serdang 43400, Selangor Darul, Malaysia. Addresses:

+ [1] Univ Putra Malaysia, Fac Engn, Dept Chem \& Environm Engn, Serdang 43400, Selangor Darul, Malaysia

+ [2] Univ Kebangsaan Malaysia, Dept Chem \& Proc Engn, Ukm Bangi 43600, Selangor, Malaysia

[ 3] Thaksin Univ, Fac Sci, Res Ctr Energy \& Environm, Phatthalung 93210, Thailand

+ [4] IIUM, Kulliyyah Engn, Dept Biotechnol Biochem Engn, POB 10, Kuala Lumpur 50728, Malaysia

E-mail Addresses: syakina@upm.edu.my

Funding

\begin{tabular}{|l|l|}
\hline Funding Agency & Grant Number \\
\hline Sime Darby Plantation, Sdn Bhd, Malaysia under the Zero Waste Technology program specifically Trust Area Biohydrogen & KK-2015-002 \\
\hline Grant Putralnisiatif Putra Muda (GP-IPM, 2018) & \\
\hline
\end{tabular}

View funding text

Publisher

ELSEVIER, RADARWEG 29, 1043 NX AMSTERDAM, NETHERLANDS

Journal Information

Impact Factor: Journal Citation Reports

\section{Categories / Classification}

Research Areas: Biotechnology \& Applied Microbiology; Engineering; Food Science \& Technology

Web of Science Categories: Biotechnology \& Applied Microbiology; Engineering, Chemical; Food Science \& Technology

\section{See more data fields}

\section{Citation Network}

In Web of Science Core Collection

1

Times Cited

Create Citation Alert

All Times Cited Counts

1 in All Databases

See more counts

\section{4}

Cited References

View Related Records

Most recently cited by:

Wang, Jianfei; Huang, Jiaqi; Guo, Huanyu; et al.

Optimization of immobilization conditions for Lactobacillus pentosus cells. BIOPROCESS AND BIOSYSTEMS ENGINEERING (2020)

View All

\section{Use in Web of Science}

Web of Science Usage Count

$\begin{array}{ll}2 & 2 \\ \text { Last } 180 \text { Days } & \text { Since } 2013 \\ \text { Learn more } & \end{array}$

\section{This record is from}

Web of Science Core Collection

- Science Citation Index Expanded

Suggest a correction

If you would like to improve the quality of the data in this record, please suggest a correction. 


\section{Cited References: 24}

1. Effect of column's temperature and evaluation of RID and ELSD as suitable ion exchange HPLC detection method of simple sugars

By: Abdul, P; Harun, S; Jahim, J. M; et al.

J. Sci. Technol. Pages: 599-604 Published: 2011

[Show additional data]

2. Overview of hydrogen production technologies from biogas and the applications in fuel cells

Times Cited: 165

By: Alves, Helton Jose; Bley Junior, Cicero; Niklevicz, Rafael Rick; et al.

INTERNATIONAL JOURNAL OF HYDROGEN ENERGY Volume: 38 Issue: 13 Pages: 5215-5225 Published: MAY 12013

3. Anaerobic biofilm reactors for dark fermentative hydrogen production from wastewater: A review

Times Cited: 48

By: Barca, Cristian; Soric, Audrey; Ranava, David; et al.

BIORESOURCE TECHNOLOGY Volume: 185 Pages: 386-398 Published: JUN 2015

4. Isolation of a new Thermoanaerobacterium thermosaccharolyticum strain (FH1) producing a thermostable dextranase

Times Cited: 26

By: Hoster, F; Daniel, R; Gottschalk, G

JOURNAL OF GENERAL AND APPLIED MICROBIOLOGY Volume: 47 Issue: 4 Pages: 187-192 Published: AUG 2001

5. Optimization of thermophilic biohydrogen production by microflora of palm oil mill effluent: cell attachment on granular activated carbon as support media

By: Jamali, NS; Jahim, J Md.

Malays J Anal Sci Volume: 20 Pages: 1437-46 Published: 2016

6. Particle size variations of activated carbon on biofilm formation in thermophilic biohydrogen production from palm oil mill effluent

Times Cited: 10

By: Jamali, Nur Syakina; Jahim, Jamaliah Md; Isahak, Wan Nor Roslam Wan; et al.

ENERGY CONVERSION AND MANAGEMENT Volume: 141 Special Issue: SI Pages: 354-366 Published: JUN 12017

7. Hydrodynamic characteristics and model of fluidized bed reactor with immobilised cells on activated carbon for biohydrogen production By: Jamali, Nur Syakina; Jahim, Jamaliah Md; O-Thong, Sompong; et al.

INTERNATIONAL JOURNAL OF HYDROGEN ENERGY Volume: 44 Issue: 18 Pages: 9256-9271 Published: APR 52019

8. Biofilm formation on granular activated carbon in xylose and glucose mixture for thermophilic biohydrogen production

By: Jamali, Nur Syakina; Jahim, Jamaliah Md; Isahak, Wan Nor Roslam Wan

INTERNATIONAL JOURNAL OF HYDROGEN ENERGY Volume: 41 Issue: 46 Pages: 21617-21627 Published: DEC 142016

9. Physicochemical characteristics of attached biofilm on granular activated carbon for thermophilic biohydrogen production

Times Cited: 15

By: Lutpi, Nabilah Aminah; Jahim, Jamaliah Md; Mumtaz, Tabassum; et al.

RSC ADVANCES Volume: 5 Issue: 25 Pages: 19382-19392 Published: 2015

10. Pretreatment conditions of palm oil mill effluent (POME) for thermophilic biohydrogen production by mixed culture

By: Mahmod, Safa Senan; Jahim, Jamaliah Md; Abdul, Peer Mohamed

INTERNATIONAL JOURNAL OF HYDROGEN ENERGY Volume: 42 Issue: 45 Special Issue: SI Pages: 27512-27522 Published: NOV 92017

Times Cited: 9

11. The Ribosomal Database Project (RDP)

Times Cited: 454

By: Maidak, BL; Olsen, GJ; Larsen, N; et al.

NUCLEIC ACIDS RESEARCH Volume: 24 Issue: 1 Pages: 82-85 Published: JAN 11996

12. Biorefineries and the food, energy, water nexus - towards a whole systems approach to design and planning

Times Cited: 21

By: Martinez-Hernandez, Elias; Samsatli, Sheila

CURRENT OPINION IN CHEMICAL ENGINEERING Volume: 18 Pages: 16-22 Published: NOV 2017

13. Acidogenic fermentation of vegetable based market waste to harness biohydrogen with simultaneous stabilization

Times Cited: 68

By: Mohan, S. Venkata; Mohanakrishna, G.; Goud, R. Kannaiah; et al.

BIORESOURCE TECHNOLOGY Volume: 100 Issue: 12 Pages: 3061-3068 Published: JUN 2009

14. Influence of support materials on continuous hydrogen production in anaerobic packed-bed reactor with immobilized hydrogen producing bacteria at

Times Cited: 12 acidic conditions

By: Muri, Petra; Marinsek-Logar, Romana; Djinovic, Petar; et al.

ENZYME AND MICROBIAL TECHNOLOGY Volume: 111 Pages: 87-96 Published: APR 2018

15. 16S rRNA-targeted probes for specific detection of Thermoanaerobacterium spp., Thermoanaerobacterium thermosaccharolyticum, and Caldicellulosiruptor spp. by fluorescent in situ hybridization in biohydrogen producing systems

By: O-Thong, Sompony; Prasertsan, Poonsuk; Karakashev, Dimitar; et al.

INTERNATIONAL JOURNAL OF HYDROGEN ENERGY Volume: 33 Issue: 21 Pages: 6082-6091 Published: NOV 2008 
17. Batch Fermentative Biohydrogen Production Process Using Immobilized Anaerobic Sludge from Organic Solid Waste

19. Application of polyethylene glycol immobilized Clostridium sp LS2 for continuous hydrogen production from palm oil mill effluent in upflow anaerobic sludge blanket reactor

By: Singh, Lakhveer; Siddiqui, Muhammad Faisal; Ahmad, Anwar; et al.

BIOCHEMICAL ENGINEERING JOURNAL Volume: 70 Pages: 158-165 Published: JAN 152013

20. Methods for enhancing bio-hydrogen production from biological process: A review

Times Cited: 88 By: Singh, Lakhveer; Wahid, Zularisam A.

JOURNAL OF INDUSTRIAL AND ENGINEERING CHEMISTRY Volume: 21 Pages: 70-80 Published: JAN 252015

21. Changes in bacterial community during fermentative hydrogen and acid production from organic waste by thermophilic anaerobic microflora By: Ueno, Y.; Sasaki, D.; Fukui, H.; et al.

JOURNAL OF APPLIED MICROBIOLOGY Volume: 101 Issue: 2 Pages: 331-343 Published: AUG 2006

22. HRT-dependent hydrogen production and bacterial community structure of mixed anaerobic microflora in suspended, granular and immobilized sludge systems using glucose as the carbon substrate

By: Wu, Shu-Yii; Hung, Chun-Hsiung; Lin, Chiu-Yue; et al.

INTERNATIONAL JOURNAL OF HYDROGEN ENERGY Volume: 33 Issue: 5 Pages: 1542-1549 Published: MAR 2008

23. EXPRESSION OF SOLVENT-FORMING ENZYMES AND ONSET OF SOLVENT PRODUCTION IN BATCH CULTURES OF CLOSTRIDIUM-BEIJERINCKII (CLOSTRIDIUM- TimES Cited: 66 BUTYLICUM)

By: YAN, RT; ZHU, CX; GOLEMBOSKI, C; et al.

APPLIED AND ENVIRONMENTAL MICROBIOLOGY Volume: 54 Issue: 3 Pages: 642-648 Published: MAR 1988

24. Succession of bacterial community and enzymatic activities of activated sludge by heat-treatment for reduction of excess sludge

Times Cited: 85 By: Yan, Sangtian; Miyanaga, Kazuhiko; Xing, Xin-Hui; et al.

BIOCHEMICAL ENGINEERING JOURNAL Volume: 39 Issue: 3 Pages: 598-603 Published: MAY 12008

\section{Showing 24 of $24 \quad$ View All in Cited References page}

